Cyclic products of the Ugi reaction of aldehydo and keto carboxylic acids: chemoselective modification

Alan Tsaloev, Alexei Ilyin, Sergey Tkachenko, Alexandre Ivachtchenko, Dmitry Kravchenko, Mikhail Krasavin*

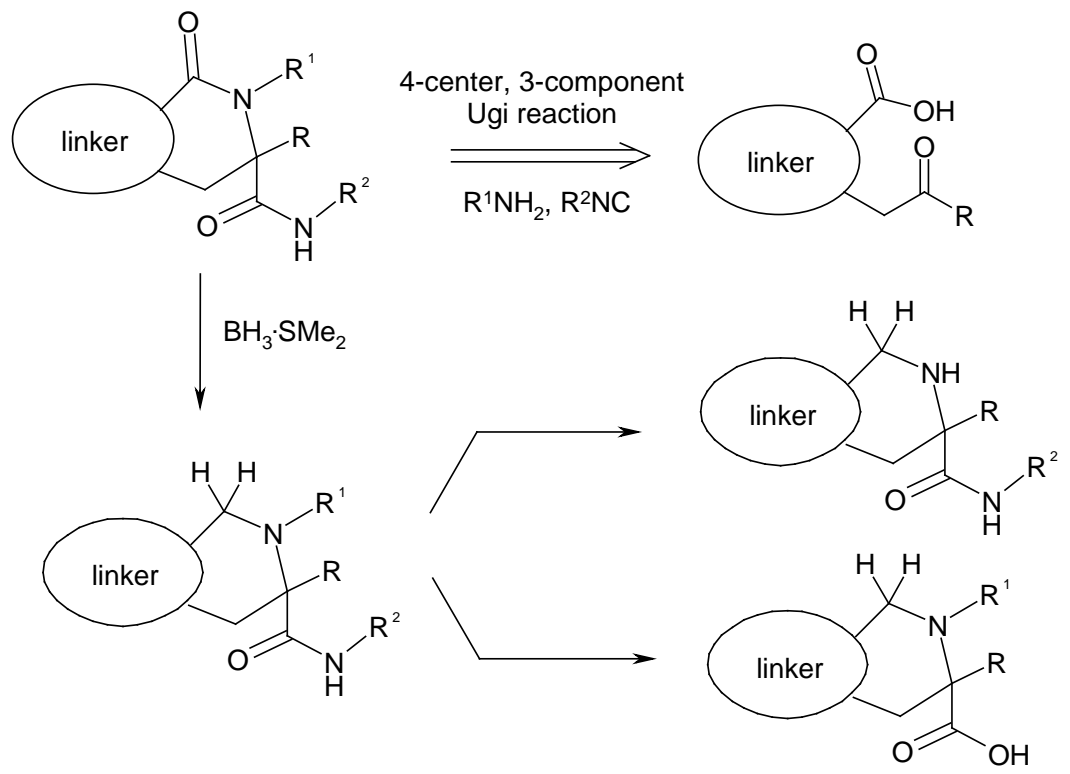




\section{Cyclic products of the Ugi reaction of aldehydo and keto carboxylic acids: chemoselective modification}

Alan Tsaloev, ${ }^{a}$ Alexei Ilyin, ${ }^{a}$ Sergey Tkachenko, ${ }^{b}$ Alexandre Ivachtchenko, ${ }^{a, b}$ Dmitry Kravchenko, ${ }^{a}$ Mikhail Krasavin* ${ }^{a, c}$

${ }^{a}$ Chemical Diversity Research Institute, $2 a$ Rabochaya St., Khimki, Moscow Reg., 141400, Russia

${ }^{b}$ ChemDiv, Inc., 6605 Nancy Ridge Drive, San Diego, CA 92121 USA

'Science and Education Center “Innovative Research”, Yaroslavl State Pedagogical University, Yaroslavl 150000, Russia

*Corresponding author, e-mail: myk@chemdiv.com, phone: +7(495)995-4944, fax: +7(495)6269780.

Abstract: A method for the chemoselective reduction of Ugi-type lactam amides at the lactam carbonyl functionality with borane complexes has been developed. The novel reduction products can be further manipulated synthetically to yield various novel $N$ - and $C$-terminally active unnatural amino acid building blocks.

Keywords: Ugi reaction, bifunctional reagents, chemoselective reduction, unnatural amino acids.

Replacement of two participants in the Ugi four-component reaction with a single bifunctional reagent has proved itself as a fruitful strategy toward various drug-like heterocycles. ${ }^{1}$ Specifically, various tethered keto (or aldehydo) carboxylic acids, when employed in the Ugi process, provide a facile (and high-yielding) entry into diverse lactam amides (Figure 1). Since the initial publications describing this methodology by Harrimann ${ }^{2}$ and $\mathrm{Ugi}^{3}$ in the late 1990s, a significant number of these peptidomimetic scaffolds have appeared in the literature. This, in 
turn, resulted in extensive investigations on the associated biological activities which has yielded promising leads for modulation of important biological targets such as androgen receptors ${ }^{4}$ and $\gamma$-secretase. ${ }^{5}$

We have been involved in identifying opportunities for chemoselective modification of these intriguing drug-like scaffolds, in pursuit of a dual goal: i) improving the physicochemical properties of lactam amides (specifically, their aqueous solubility); and ii) preparing functionalized peptidomimetic building blocks based on these scaffolds which are useful in designing yet more novel compound libraries for biological screening. Herein, we report several examples of efficient, chemoselective reduction of such lactam amides and further transformation of the resulting cyclic tertiary amines into $\mathrm{N}$ - and $\mathrm{C}$-terminally active unnatural amino acid building blocks.

A set of 22 3-oxo-2,3,4,5-tetrahydro-1,4-benzoxazepine-5-carboxamides 1a-x (Scheme 1) was prepared in good to excellent yields from the known 2-(2-formylphenoxy)acetic acid ${ }^{6}$ using various amines and two isocyanides as described earlier. ${ }^{7}$ Inspired by the successful application of borane complexes toward reduction of linear Ugi reaction derived diamides, as recently disclosed by Tron, $^{8}$ we exposed lactam amides $1 \mathbf{a}-\mathbf{x}$ to an excess amount of borane-dimethyl sulfide complex in THF at room temperature (Scheme 1). To our delight, in all cases, this led to a clean and complete reduction of the lactam function only, with no trace of secondary amide reduction, even on prolonged (3 days) treatment at room temperature (heating of selected reaction mixtures to reflux ultimately led to the accumulation of the double-reduction material, however, this was never characterized due to difficulties in its isolation). The stable tertiary amine-borane complex immediately resulting from such reductions was destroyed by briefly refluxing the crude reaction mixture in saturated methanolic $\mathrm{HCl}$. The product 2,3,4,5tetrahydro-1,4-benzoxazepine-5-carboxamides $\mathbf{6 a - x}$ were isolated chromatographically in good to excellent yields (Table 1). A notable feature of these hitherto unknown compounds $\mathbf{6}$ is that they are close shape mimics of the peptidomimetic lactam amides $\mathbf{1}$ containing a basic nitrogen. 
Hence, they are expected to have better aqueous solubility and the basic center can be utilized for the preparation of various salts (including diastereomeric salts for enantiomer resolution) - both features having much appeal for the development of drug candidates based on this novel scaffold. Moreover, the utility of this chemoselective lactam reduction procedure can be extended to other lactam amides (3 and 5), also prepared via the Ugi reaction of the keto acids 2 and 4, respectively. The yield from the reduction of aromatic lactam 5 was low (45 \%) while the aliphatic lactam 3 was reduced in an excellent 84 \% yield (Scheme 2).

The nature of the borane reducing agent seems unimportant for the outcome of the lactam reduction. For example, borane-THF complex generated in situ from sodium borohydride and iodine $^{9}$ reduces selected lactams 1 in yields similar to those obtained using the $\mathrm{BH}_{3} \cdot \mathrm{SMe}_{2}$ procedure (Scheme 3).

Compounds 6 are, essentially, amides of an unnatural $\alpha$-amino acid. We were successful in removing a benzyl group from the compounds $\mathbf{6 k}$ and $\mathbf{6 w}$ (with remarkable chemoselectivity with respect to the second benzyl group in the latter case) and exposing the secondary nitrogen atom in the resulting products 9a,b to further modifications (for example, efficient reductive alkylation with aldehydes or ketones, Scheme 4). Likewise, selected tert-butyl amides 6c and $\mathbf{6 e}(\mathbf{6 k})$, when treated with strong Brønsted acids at reflux, furnished nearly quantitative yields of the novel cyclic $\alpha$-amino acids 11a-c, without disruption of the tetrahydro-1,4-benzoxazepine cycle (Scheme 5).

In summary, we have described some new evolutionary directions for the Ugi reaction derived lactam amides to be developed into novel, drug-like heterocyclic scaffolds and unnatural amino acid building blocks.

\section{Experimental Section}


General Procedure 1: Synthesis of lactam amides 1 (3 or 5). The aldehydo or keto carboxylic acid (1.0 eq.) and primary amine (1.0 eq.) were dissolved in $\mathrm{MeOH}$. The solution was stirred at $\mathrm{r}$. t. for 10 min. The appropriate isocyanide (1.2 eq.) was added and the resulting mixture was stirred at $50{ }^{\circ} \mathrm{C}$ for $2-3 \mathrm{~h}$. On completion, the reaction mixture was cooled to r.t., and the resulting precipitate was filtered, washed with $\mathrm{MeOH}$, and purified by crystallization from $\mathrm{Et}_{2} \mathrm{O}$ of by chromatography on silica gel, using an appropriate gradient of $\mathrm{MeOH}$ in $\mathrm{CH}_{2} \mathrm{Cl}_{2}$ as eluent.

1a: White solid, mp $=130-132{ }^{\circ} \mathrm{C} .{ }^{1} \mathrm{H}$ NMR (400 MHz, DMSO- $\left.d_{6}\right): \delta 7.45(\mathrm{~d}, J=7.6 \mathrm{~Hz}, 1 \mathrm{H})$, $7.24(\mathrm{t}, J=7.6 \mathrm{~Hz}, 1 \mathrm{H}), 7.07-7.05(\mathrm{~m}, 2 \mathrm{H}), 6.94(\mathrm{~d}, J=8.0 \mathrm{~Hz}, 1 \mathrm{H}), 5.09$ (d, $J=14.4 \mathrm{~Hz}, 1 \mathrm{H})$, 4.99 (s, 1H), 4.19 (d, $J=14.4$ Hz, 1H), 3.57 (m, 1H), 3.15 - 3.11 (m, 1H), 1.55 - 1.52 (m, 2H), 1.23 (s, 9H), 0.81 (t, $J=7.6 \mathrm{~Hz}, 3 \mathrm{H}) .{ }^{13} \mathrm{C}$ NMR (100 MHz, DMSO-d $): \delta 169.5,168.1,156.4$, 130.3, 129.2, 127.5, 122.8, 119.9, 72.4, 63.9, 50.6, 49.9, 28.1, 20.5, 11.0. Anal. Calcd for $\mathrm{C}_{17} \mathrm{H}_{24} \mathrm{~N}_{2} \mathrm{O}_{3}$ : C, 67.08; H, 7.95; N, 9.20. Found: C, 67.14; H, 8.01; N, 9.23.

General Procedure 2: Reduction of lactam amides 1 (3 or 5). A solution of 1 (3 or 5) (1 mmol) in anhydrous THF (3 mL) was treated with $\mathrm{BH}_{3} \cdot \mathrm{SMe}_{2}$ in THF (2 M solution, $2 \mathrm{~mL}, 4$ mmol). This mixture was stirred overnight at r.t. The solvent was evaporated and the residue was dissolved in sat. $\mathrm{MeOH}-\mathrm{HCl}$. The solution was heated at reflux for $30 \mathrm{~min}$ and after cooling to r.t., was neutralized with $10 \%$ aq. $\mathrm{K}_{2} \mathrm{CO}_{3}$, and the resulting neutral solution was extracted with $\mathrm{CH}_{2} \mathrm{Cl}_{2}$ (3 x $50 \mathrm{~mL}$ ). The combined organic extract was dried over anhydrous $\mathrm{MgSO}_{4}$, filtered and concentrated in vacuo. The residue was purified by preparative TLC (silica gel, $\mathrm{CH}_{2} \mathrm{Cl}_{2}$ ).

6a: Waxy-beige solid. ${ }^{1} \mathrm{H}$ NMR (400 MHz, $\left.\mathrm{CDCl}_{3}\right)$ : $\delta 7.32(\mathrm{~s}, 1 \mathrm{H}), 7.24-7.17(\mathrm{~m}, 2 \mathrm{H}), 7.03$ (t, $J=10.0 \mathrm{~Hz}, 1 \mathrm{H}), 6.97$ (d, $J=10.8 \mathrm{~Hz}, 1 \mathrm{H}), 4.27$ (s, 1H), 4.09 - 3.98 (m, 2H), 3.12 - 3.04 (m, 1H), 2.90 - $2.82(\mathrm{~m}, 1 \mathrm{H}), 2.48(\mathrm{t}, J=8.0 \mathrm{~Hz}, 2 \mathrm{H}), 1.52(\mathrm{~m}, 2 \mathrm{H}), 1.37$ (s, 9H), 0.92 (t, $J=9.6 \mathrm{~Hz}$, 3H). ${ }^{13} \mathrm{C}$ NMR (100 MHz, $\left.\mathrm{CDCl}_{3}\right): \delta 169.7,155.8,130.8,128.7,123.1,120.7,70.0,67.4,54.9$, 50.1, 49.6, 28.2, 20.4, 11.2. Anal. Calcd for $\mathrm{C}_{17} \mathrm{H}_{26} \mathrm{~N}_{2} \mathrm{O}_{2}$ : C, 70.31; H, 9.02; N, 9.65. Found: C, 70.28; H, 8.92; N, 9.55. 
Acknowledgements. This research was supported by the Federal Agency for Science and Innovation (Russian Federation Government Contract 02.740.11.0092). Dr. Alex Khvat and Dr. Volodymyr Kysil of ChemDiv, Inc. (San Diego, USA) are acknowledged for helpful discussions regarding the strategies for lactam reduction.

Supporting Information: Characterization data and preparative procedures for the newly synthesized compounds (1a-x, 6a-x, 3, 5, 7, 8, 9a-b, 10a-d, 11a-c).

\section{References}

1. For an excellent review of applications of bifunctional reagents in the Ugi reaction, see: Hulme, C.; Dietrich, J. Mol. Diversity 2009, 13, 195-207 - and references cited therein.

2. Harriman, G. C. B. Tetrahedron Lett. 1997, 38, 5591-5594.

3. Hanusch-Kompa, C.; Ugi, I. Tetrahedron Lett. 1998, 39, 2725-2728.

4. Rafferty, S. W.; Stewart, E. L.; Turnbull, P. S.; Yates, C. M. PCT Int. Appl. WO2008121602; Chem. Abstr. 2008, 149, 425988.

5. Galley, G.; Goodnow, R. A.; Peters, J.-U. US Pat. Appl. Publ. US2004235819; Chem. Abstr. 2004, 142, 6564.

6. Hullar, T. L.; Failla, D. L. J. Med. Chem. 1969, 12, 420-424.

7. Ilyin, A. P.; Parchinsky, V. Z.; Peregudova, J. N.; Trifilenkov, A. S.; Poutsykina, E. B.; Tkachenko, S. E.; Kravchenko, D. V.; Ivachtchenko, A. V. Tetrahedron Lett. 2006, 47, 26492653.

8. Pirali, T.; Callipari, G.; Ercolano, E.; Genazzani, A. A.; Giovenzana, G. B.; Tron, G. C. Org. Lett. 2008, 10, 4199-4202.

9. Prasad, A. S. B.; Kanth, J. V. B.; Periasamy, M. Tetrahedron Lett. 1992, 48, 4623-4628. 
Figure 1. Cyclic lactam amides resulting from the Ugi reaction of bifunctional keto (aldehydo) carboxylic acids.

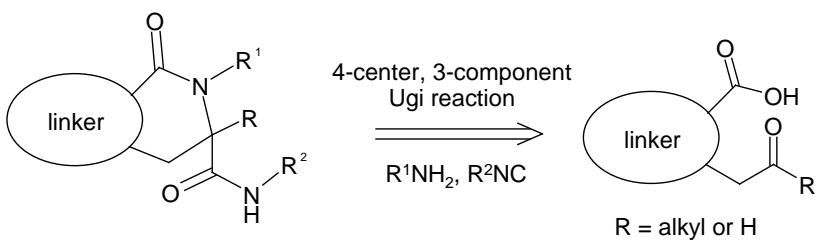

Scheme 1. Ugi reaction of 2-(2-formylphenoxy)acetic acid and chemoselective reduction of the resulting lactam amides $\mathbf{1 a}-\mathbf{x}$.
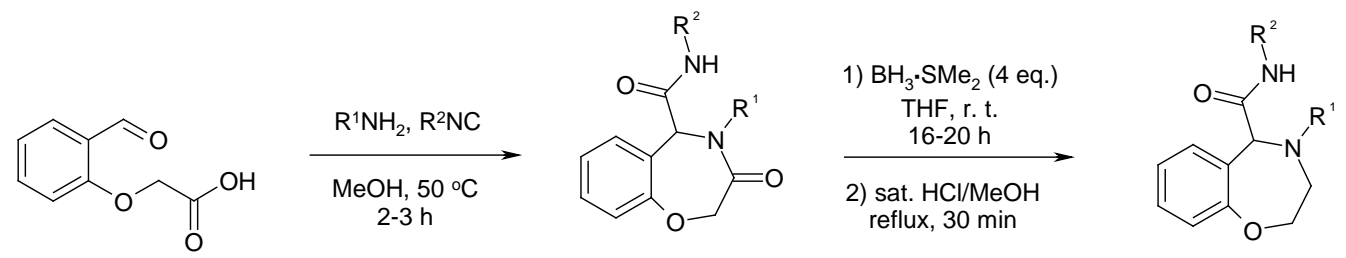

1a-x

Scheme 2. Preparation and chemoselective reduction of lactam amides $\mathbf{3}$ and $\mathbf{5 .}$
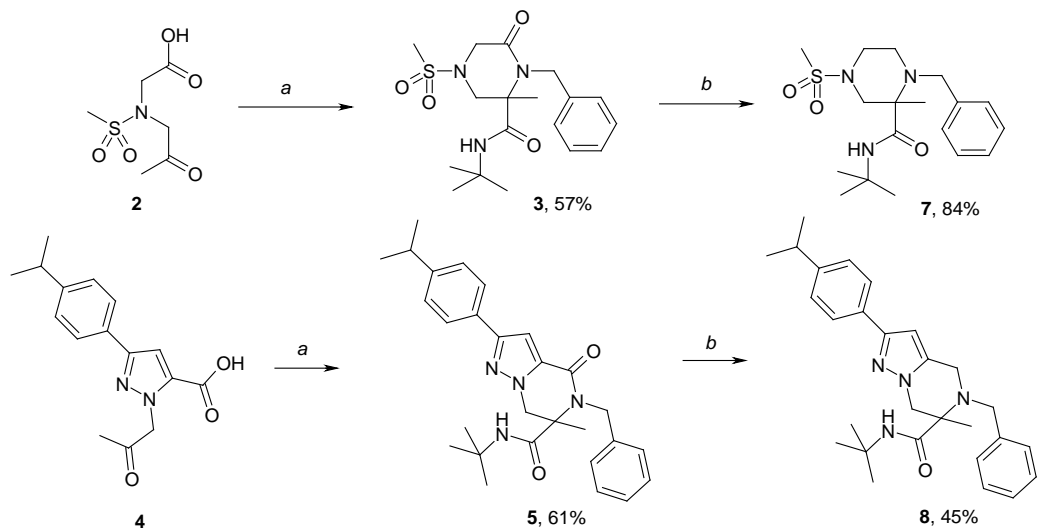

Reagents and conditions: (a) $t$ - $\mathrm{BuNC}$ (1.2 eq.), $\mathrm{BnNH}_{2}$ (1.0 eq.), $\mathrm{MeOH}, 50{ }^{\circ} \mathrm{C}, 3 \mathrm{~h}$; (b) $\mathrm{BH}_{3} \cdot \mathrm{SMe}_{2}(4.0$ eq.), THF, r. t. 18 h; sat. $\mathrm{MeOH}-\mathrm{HCl}$, reflux, $30 \mathrm{~min}$.

Scheme 3. Chemoselective reduction of the selected lactam amides $\mathbf{1}$ using the $\mathrm{NaBH}_{4}-\mathrm{I}_{2}$ protocol.
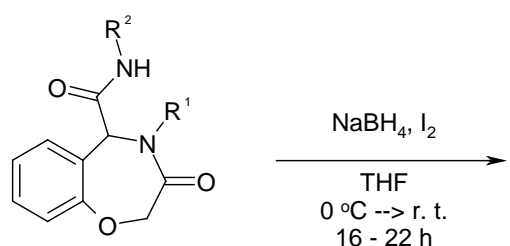

1

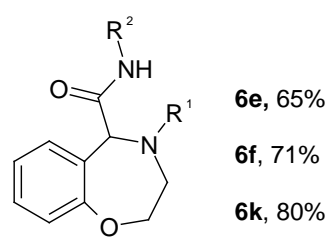

6k, $80 \%$ 
Scheme 4. $N$-Debenzylation of compounds $6 \mathbf{k}$ and $6 \mathbf{w}$ and reductive alkylation of the resulting amines.

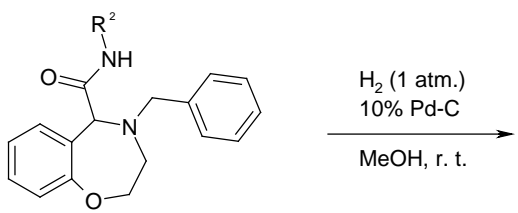

$6 k, 6 w$

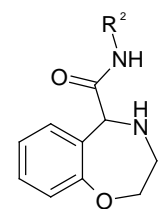

$9 a, R^{2}=t-B u, 88 \%$

9b, $\mathrm{R}^{2}=4-\mathrm{CF}_{3} \mathrm{C}_{6} \mathrm{H}_{4} \mathrm{CH}_{2}, 94 \%$

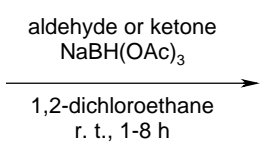

r. t., 1-8 $\mathrm{h}$

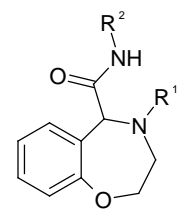

10a, $\mathrm{R}^{2}=t-\mathrm{Bu}, \mathrm{R}^{1}=4-\mathrm{FC}_{6} \mathrm{H}_{4} \mathrm{CH}_{2}, 74 \%$

10b, $\mathrm{R}^{2}=t$-Bu, $\mathrm{R}^{1}=1$-acetylpiperidin-4-yl, 55\% 10c, $\mathrm{R}^{2}=t$-Bu, $\mathrm{R}^{1}=1$-Boc-piperidin-4-yl, $53 \%$

10d, $\mathrm{R}^{2}=4-\mathrm{CF}_{3} \mathrm{C}_{6} \mathrm{H}_{4} \mathrm{CH}_{2}, \mathrm{R}^{1}=4-\mathrm{FC}_{6} \mathrm{H}_{4} \mathrm{CH}_{2}, 66 \%$

6b, $\quad \mathrm{R}^{2}=t-\mathrm{Bu}, \mathrm{R}^{1}=i-\mathrm{Pr}, 69 \%$

6n, $\mathrm{R}^{2}=4-\mathrm{CF}_{3} \mathrm{C}_{6} \mathrm{H}_{4} \mathrm{CH}_{2}, \mathrm{R}^{1}=i-\mathrm{Pr}, 78 \%$

Scheme 5. Tert-butyl amide hydrolysis.<smiles>CC(C)CN1CCOc2ccccc2C1C(=O)NC(C)(C)C</smiles>

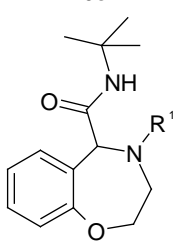

$6 e, 6 k$
$48 \% \mathrm{HBr}$ in $\mathrm{AcOH}$

$130{ }^{\circ} \mathrm{C}, 16 \mathrm{~h}$

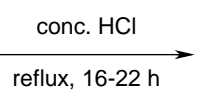

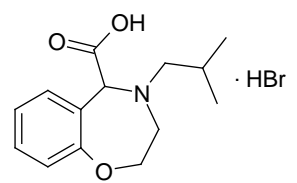

$11 a, 98 \%$

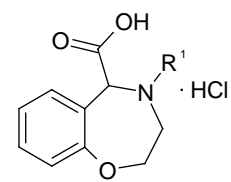

11b, $\mathrm{R}^{1}=$ cyclopropyl, $96 \%$

11c. $\mathrm{R}^{1}=$ benzyl, $98 \%$ 
Table 1. Preparation and chemoselective reduction of the lactam amides 1a-x (Scheme 1).

\begin{tabular}{|c|c|c|c|c|c|c|}
\hline Entry & $\mathrm{R}^{1}$ & $\mathrm{R}^{2}$ & $\begin{array}{l}\text { Yield of 1, } \\
(\%)\end{array}$ & $\begin{array}{c}\text { LC MS } \\
{\left[\mathrm{M}+\mathrm{H}^{+}\right]} \\
\mathrm{m} / \mathrm{z}\end{array}$ & $\begin{array}{l}\text { Yield of } 6, \\
(\%)\end{array}$ & $\begin{array}{c}\text { LC MS } \\
{\left[\mathrm{M}+\mathrm{H}^{+}\right]} \\
\mathrm{m} / \mathrm{z}\end{array}$ \\
\hline 1(6)a & $n-\operatorname{Pr}$ & $t$-Bu & 60 & 305.6 & 78 & 291.4 \\
\hline 1(6)b & $i-\operatorname{Pr}$ & $t$-Bu & 64 & 305.7 & 50 & 291.3 \\
\hline 1(6)c & $i$-Bu & $t-\mathrm{Bu}$ & 51 & 319.7 & 53 & 305.7 \\
\hline 1(6)d & $\mathrm{EtO}\left(\mathrm{CH}_{2}\right)_{3}$ & $t-\mathrm{Bu}$ & 68 & 349.2 & 56 & 335.6 \\
\hline 1(6)e & cyclopropyl & $t-\mathrm{Bu}$ & 86 & 303.5 & 81 & 289.5 \\
\hline 1(6)f & cyclopentyl & $t-\mathrm{Bu}$ & 74 & 331.4 & 74 & 317.2 \\
\hline 1(6)g & cycloheptyl & $t$-Bu & 66 & 359.8 & 63 & 345.8 \\
\hline 1(6)h & $\mathrm{Ph}\left(\mathrm{CH}_{2}\right)_{2}$ & $t$-Bu & 46 & 367.1 & 57 & 353.7 \\
\hline 1(6)i & 4- $\mathrm{MeC}_{6} \mathrm{H}_{4}\left(\mathrm{CH}_{2}\right)_{2}$ & $t-\mathrm{Bu}$ & 54 & 381.6 & 63 & 367.7 \\
\hline $1(6) j$ & $\begin{array}{c}3,4- \\
(\mathrm{MeO})_{2} \mathrm{C}_{6} \mathrm{H}_{3}\left(\mathrm{CH}_{2}\right)_{2}\end{array}$ & $t-\mathrm{Bu}$ & 56 & 427.5 & 70 & 413.2 \\
\hline $1(6) k$ & $\mathrm{Bn}$ & $t-\mathrm{Bu}$ & 72 & 353.5 & 82 & 339.5 \\
\hline 1(6)I & 4- $\mathrm{ClC}_{6} \mathrm{H}_{4} \mathrm{CH}_{2}$ & $t-\mathrm{Bu}$ & 66 & 387.5 & 51 & 373.8 \\
\hline 1(6)m & $n-\operatorname{Pr}$ & $4-\mathrm{CF}_{3} \mathrm{C}_{6} \mathrm{H}_{4} \mathrm{CH}_{2}$ & 67 & 407.5 & 71 & 393.6 \\
\hline $1(6) n$ & $i-\operatorname{Pr}$ & $4-\mathrm{CF}_{3} \mathrm{C}_{6} \mathrm{H}_{4} \mathrm{CH}_{2}$ & 52 & 407.5 & 89 & 393.3 \\
\hline 1(6)o & $i$-Bu & $4-\mathrm{CF}_{3} \mathrm{C}_{6} \mathrm{H}_{4} \mathrm{CH}_{2}$ & 43 & 420.6 & 93 & 407.6 \\
\hline 1(6)p & $\mathrm{EtO}\left(\mathrm{CH}_{2}\right)_{3}$ & $4-\mathrm{CF}_{3} \mathrm{C}_{6} \mathrm{H}_{4} \mathrm{CH}_{2}$ & 74 & 451.3 & 65 & 437.6 \\
\hline $1(6) q$ & cyclopropyl & $4-\mathrm{CF}_{3} \mathrm{C}_{6} \mathrm{H}_{4} \mathrm{CH}_{2}$ & 86 & 405.6 & 94 & 391.5 \\
\hline $1(6) r$ & cyclopentyl & $4-\mathrm{CF}_{3} \mathrm{C}_{6} \mathrm{H}_{4} \mathrm{CH}_{2}$ & 58 & 433.3 & 67 & 419.5 \\
\hline 1(6)s & cycloheptyl & $4-\mathrm{CF}_{3} \mathrm{C}_{6} \mathrm{H}_{4} \mathrm{CH}_{2}$ & 74 & 461.7 & 54 & 447.7 \\
\hline $1(6) t$ & $\mathrm{Ph}\left(\mathrm{CH}_{2}\right)_{2}$ & $4-\mathrm{CF}_{3} \mathrm{C}_{6} \mathrm{H}_{4} \mathrm{CH}_{2}$ & 69 & 469.4 & 67 & 455.6 \\
\hline 1(6)u & 4- $\mathrm{MeC}_{6} \mathrm{H}_{4}\left(\mathrm{CH}_{2}\right)_{2}$ & $4-\mathrm{CF}_{3} \mathrm{C}_{6} \mathrm{H}_{4} \mathrm{CH}_{2}$ & 55 & 483.5 & 65 & 469.6 \\
\hline $1(6) v$ & $\begin{array}{c}\stackrel{3,4-}{(\mathrm{MeO})}{ }_{2} \mathrm{C}_{6} \mathrm{H}_{3}\left(\mathrm{CH}_{2}\right)_{2}\end{array}$ & $4-\mathrm{CF}_{3} \mathrm{C}_{6} \mathrm{H}_{4} \mathrm{CH}_{2}$ & 82 & 529.5 & 75 & 515.4 \\
\hline $1(6) w$ & $\mathrm{Bn}$ & $4-\mathrm{CF}_{3} \mathrm{C}_{6} \mathrm{H}_{4} \mathrm{CH}_{2}$ & 79 & 455.6 & 56 & 441.7 \\
\hline $1(6) x$ & $4-\mathrm{ClC}_{6} \mathrm{H}_{4} \mathrm{CH}_{2}$ & $4-\mathrm{CF}_{3} \mathrm{C}_{6} \mathrm{H}_{4} \mathrm{CH}_{2}$ & 47 & 489.6 & 71 & 475.9 \\
\hline
\end{tabular}

